\title{
ESTRESORES EMOCIONALES DE ESTUDIANTES DE ENFERMERÍA EN PRÁCTICAS CLÍNICAS INTRAHOSPITALARIAS
}

\author{
INFIRMARY STUDENTS' EMOTIONAL ESTRESSORS IN
} INTRAHOSPITAL CLINICAL PRACTICE

\author{
Edith Morales H. ${ }^{1}$, Claudia Amigo R. ${ }^{1}$, Alejandra J. Álvarez H. ${ }^{2}$, Miguel A. Bustamante U. ${ }^{2 *}$
}

1 Facultad de Ciencias de la Salud, Universidad de Talca, Dos Norte 685, 3465548 Talca, Chile. E-mail: ale.alvarez.hor@gmail.com 2 Facultad de Economía y Negocios, Universidad de Talca, Dos Norte 685, 3465548 Talca, Chile. E-mail:: mabu@utalca.cl

RESUMEN

ABSTRACT

Este trabajo estudia la presencia de estresores en estudiantes de enfermería de tercer año durante la realización de sus prácticas clínicas intrahospitalarias. Se aplicó una metodología cuantitativa por medio del cuestionario bilingüe Kezkak, del cual se analizaron las dimensiones, falta de competencia, sobrecarga, relación con tutores y compañeros e implicación emocional. Se realizó un piloteo del instrumento con docentes y estudiantes de la escuela de enfermería para verificar entendimiento y comprensión del mismo. Los estudiantes fueron contactados vía on-line lográndose una cobertura de $89,6 \%$. Concluyéndose finalmente que los estudiantes de enfermería presentaron estrés durante las prácticas clínicas intrahospitalarias preferentemente al inicio de estas.

PALABRAS CLAVES: Prácticas clínicas, estudiantes de enfermería, estresores, estrés.
This paper studies the presence of stressors in third-year nursing students during the performance of their intrahospital clinical practices. A quantitative methodology was applied through the Kezkak bilingual questionnaire, from which the dimensions were analyzed; Lack of competence, overload, relationship with tutors and peers and emotional involvement. The instrument was piloted with teachers and students from the nursing school to verify understanding and understanding of it. The students were contacted via on-line obtaining coverage of $89.6 \%$. Finally it was concluded that nursing students presented stress during intrahospital clinical practices, preferably at the beginning of these.

KEY WORDS: Clinical practices, infirmary students, stressors, stress. 


\section{INTRODUCCIÓN}

El estrés es una respuesta no específica del cuerpo ante cualquier demanda sobre él (Selye, 1976), siendo esto, una consecuencia de la rapidez con que las personas viven cada momento (Bruno Selye, 1975). Sus diversas interpretaciones le han asignado un entorno complejo conformado por elementos biológicos, situacionales, sociales, psicológicos, entre muchos otros (Cannon, 1932; Holmes y Rahe, 1967; Selye,1960; Lazarus y Folkman,1984), llegando a la conclusión que su determinante critico se basa en cómo la persona percibe y responde a los acontecimientos que enfrenta (Lazarus y Folkman, 1984).

Visto desde la perspectiva laboral (Da Silva, 2006), los determinantes críticos del estrés (Celis, et al., 2001) se potencian debido a la constante competencia y relaciones internas preocupantes (Davis y Newstrom, 1993), sobretodo en áreas en donde trabajadores y/o funcionarios (González, 2017) deben responder a las necesidades del servicio que ofrecen (Gil-Lacruz, 2009).

Bajo esta lógica, quienes se desarrollan en áreas de la salud (González, 2017), son vulnerables a presentar altísimas probabilidades de cuadros de estrés dadas las condiciones y desarrollo de la profesión y su constante contacto con el público, afectando su calidad de vida laboral (Granados, 2011). En estos casos, el estrés se presenta antes de entrar al mundo laboral (Burgos, 2004), es decir, se inicia durante el periodo formativo, en donde la etapa de transición desde la Universidad al mundo laboral es bastante compleja (Celis, et al., 2001), lo cual genera grandes fuentes de tensión entre los futuros profesionales (Gil-Lacruz y García-Moran, 2016).

Lo anterior puede verse reflejado en diversos estudios realizados por Zryewskyj y Davis (1987) quienes demostraron la presencia de estrés en estudiantes de enfermería durante sus prácticas clínicas. Así mismo Mahat (1996), estudiando los estresores que afectan al ámbito clínico, indicó que el 50\% de los casos tenían relación con el área interpersonal (López y López, 2011), aspecto conductual que en la formación implica potenciar el componente teórico y práctico de los alum- nos de enfermería (Collados, 2012).

Se debe tener en cuenta que los alumnos de enfermería por las características de la profesión están en contacto con el sufrimiento, dolor, invalidez e incluso la muerte (López et al., 2011), así como en estrecha interrelación con otros profesionales (Granados, 2011, también expuestos a diversos estresores (Zryewsky y Davis, 1987).

Se destaca en algunos estudios que, factores como, la falta de competencia, impotencia e incertidumbre, contacto con el sufrimiento y riesgo de dañarse en la relación con el enfermo (Cobo, Carbonell, Rodríguez, Vivo, Castellanos y Sánchez, 2010, pp.3), incrementan la percepción de estrés durante las prácticas clínicas.

De forma similar, estudios realizados con profesionales de enfermería (González, 2017), muestran que se impacta la calidad de vida laboral y que, algunas afectaciones provienen del estrés que se hace evidente a lo largo de la vida profesional (Granados, 2011) y que, en el sector de la salud, se manifiesta a causa del "contacto con el sufrimiento", "impotencia e incertidumbre", "implicación emocional", "no controlar la relación con el enfermo", "resultar dañado en la relación con el enfermo", "relación con compañeros" y "sobrecarga laboral" (Zupiria-Gorostidi., 2006).

López y Sánchez (2005) refieren que, entre las situaciones vividas con más intensidad por los estudiantes de enfermería durante las prácticas asistenciales, se encuentran: la muerte del paciente, el sufrimiento, el contacto con enfermos terminales y el afrontamiento de la muerte, las situaciones de patología crítica, el paro cardiorrespiratorio, la relación con el enfermo y su familia y la diferencia entre la teoría y la práctica. No obstante, las relaciones con los profesionales (González, 2017), los sentimientos de impotencia y la falta de conocimientos y destreza en las técnicas, afectan la calidad de vida laboral (Burgos, 2004) y son además, los aspectos que más preocupan a los alumnos de enfermería (Celis, et al., 2001).

Por su parte Timmins y Kaliszer (2002) indican que las principales fuentes de estrés en tercero de enfermería son: el estrés aca- 
démico, la relación con los profesores, las relaciones en las prácticas clínicas, las finanzas y la muerte de los pacientes (Zupiria-Gorostidi, 2006).

Complementariamente, López y López (2011), indican que la práctica clínica permite a los alumnos comparar conocimientos teóricos adquiridos con los de la práctica habitual, por una parte, para desarrollar habilidades y actitudes propias de los profesionales sanitarios (Cobo, et al, 2010) y, por otra, porque se habilitan para enfrentar un nuevo rol para el que aún no se sienten completamente preparado y una de cuyas soluciones sería incrementar la duración de la carrera asociada al desarrollo de especialidades en enfermería (Pérez, Alameda y Albéniz, 2002).

Sobre la base de lo indicado, el presente estudio busca determinar las principales causas de estrés de los estudiantes de tercer año de la carrera de enfermería de una Universidad pública, en relación a las prácticas clínicas realizadas en el área intrahospitalaria.

\section{METODOLOGÍA}

El estudio fue no experimental, cuantitativo, correlacional y de asociación de estresores laborales para analizar la evolución de las variables debidamente operacionalizadas.

\section{INSTRUMENTO DE MEDICIÓN}

El instrumento utilizado fue el cuestionario bilingüe de estresores de los estudiantes de enfermería en las prácticas clínicas, Kezkak, (2002), creado por investigadores del Departamento Enfermería II; Escuela Universitaria de Enfermería de Donostia-San Sebastián, Universidad del País Vasco, San Sebastián, España (Zupiria, et al. 2002).

El mencionado cuestionario Kezkak ha sido validado tanto a nivel internacional como nacional en diferentes áreas de estudio, incluyendo el área de docencia (Gac Sanit 2003; 17(1):37-51).

El instrumento mide los estresores a partir de 50 ítems que reflejan distintas situaciones potencialmente estresantes a las que pueden enfrentarse los estudiantes en el transcurso de sus prácticas clínicas (Tabla 1). Para cada ítem se establecen cuatro (4) respuestas en una escala tipo Likert (1932) donde $(0)=$ nada; $(1)=\operatorname{algo},(2)=$ bastante y (3) $=$ mucho. Se consideró estrés a todos aquellos ítems que obtuvieron una puntuación media superior a 1,5 .

Tabla 1: Descripción de reactivos independientes o ámbitos de estrés

\begin{tabular}{ll}
\hline $\begin{array}{l}\text { Relación con tutores y } \\
\text { compañeros, }\end{array}$ & $\begin{array}{l}\text { Se ocupa de la relación que se establezca con los profesionales y tutor del centro } \\
\left.\text { de práctica (preguntas } \mathrm{N}^{\circ} 12,28,19,25,1,20\right) .\end{array}$ \\
\hline Implicación emocional, & $\begin{array}{l}\text { Conlleva el hecho que se establezca una relación más allá de lo estrictamente } \\
\left.\text { profesional (preguntas } \mathrm{N}^{\circ} 21,8,22,31\right) .\end{array}$ \\
\hline Falta de competencia, & $\begin{array}{l}\text { Se entiende como no tener la capacidad de hacer bien el trabajo y perjudicar al } \\
\text { paciente y posiblemente al que da la atención (preguntas } \mathrm{N}^{\circ} 15,16,13,4,2,6,26,1, \\
\\
3,17,5) .\end{array}$ \\
\hline Sobrecarga, & $\begin{array}{l}\text { Entendido como exceso de trabajo y de horario de prácticas (preguntas } \mathrm{N}^{\circ} 35,36, \\
34,30,31)\end{array}$ \\
\hline
\end{tabular}

El cuestionario recoge además datos demográficos tales como edad, sexo, procedencia, estudios previos de pregrado, situación económica, antecedentes académicos (reprobación y posesión de alguna beca relacionada con su desempeño académico).

En lo sustancial, recoge la percepción de los estudiantes respecto de algún proble- ma del ánimo, sentimiento de estrés, a que atribuye sus percepciones, si ha tenido algún problema con algún paciente, que lo identifique y que, finalmente, lo explique brevemente.

PROCEDIMIENTO

La muestra estuvo constituida por un cen- 
so (Hernández et al., 2006) realizado a los estudiantes de la carrera de enfermería que cursaban tercer año y que realizaron prácticas clínicas intrahospitalarias de 20 días en el servicio de medicina y cirugía durante el semestre primavera 2016.

A su vez, para asegurar la aplicación de las normas de ética en las investigaciones, se optó por aplicar el cuestionario en forma anónima bajo el principio de consentimiento informado (Eyssautier, 2006).

El análisis de los datos se realizó resguardando la identidad, tratando de manera agrupada y con absoluta reserva la información de los respondientes.

\section{ANÁLISIS DE LA INFORMACIÓN}

Los estresores laborales fueron identificados de entre los sucesos o situaciones relacionadas con los trabajos asignados a los estudiantes en sus prácticas profesionales (Kezkak, 2002), considerando los procedimientos adecuados de acceso a la información (Zupiria, et al. 2002) y que situaciones de contexto (García-Moran y Gil-Lacruz, 2016) pueden provocar una respuesta de estrés en la persona evaluada pero sin modificar conductas o alterar el entorno en el cual se desarrollaron.

El software estadístico utilizado para el análisis de datos fue el sistema informático Statistical Package for the Social Sciences (SPSS) 15, con el que se realizó análisis descriptivo de frecuencias de las variables involucradas en el análisis.

\section{RESULTADOS}

El análisis descriptivo muestra los datos demográficos y académicos de los estudiantes analizados los que se sintetizan en la Tabla 2.

Tabla 2: Información demográfica y de antecedentes estudiantiles

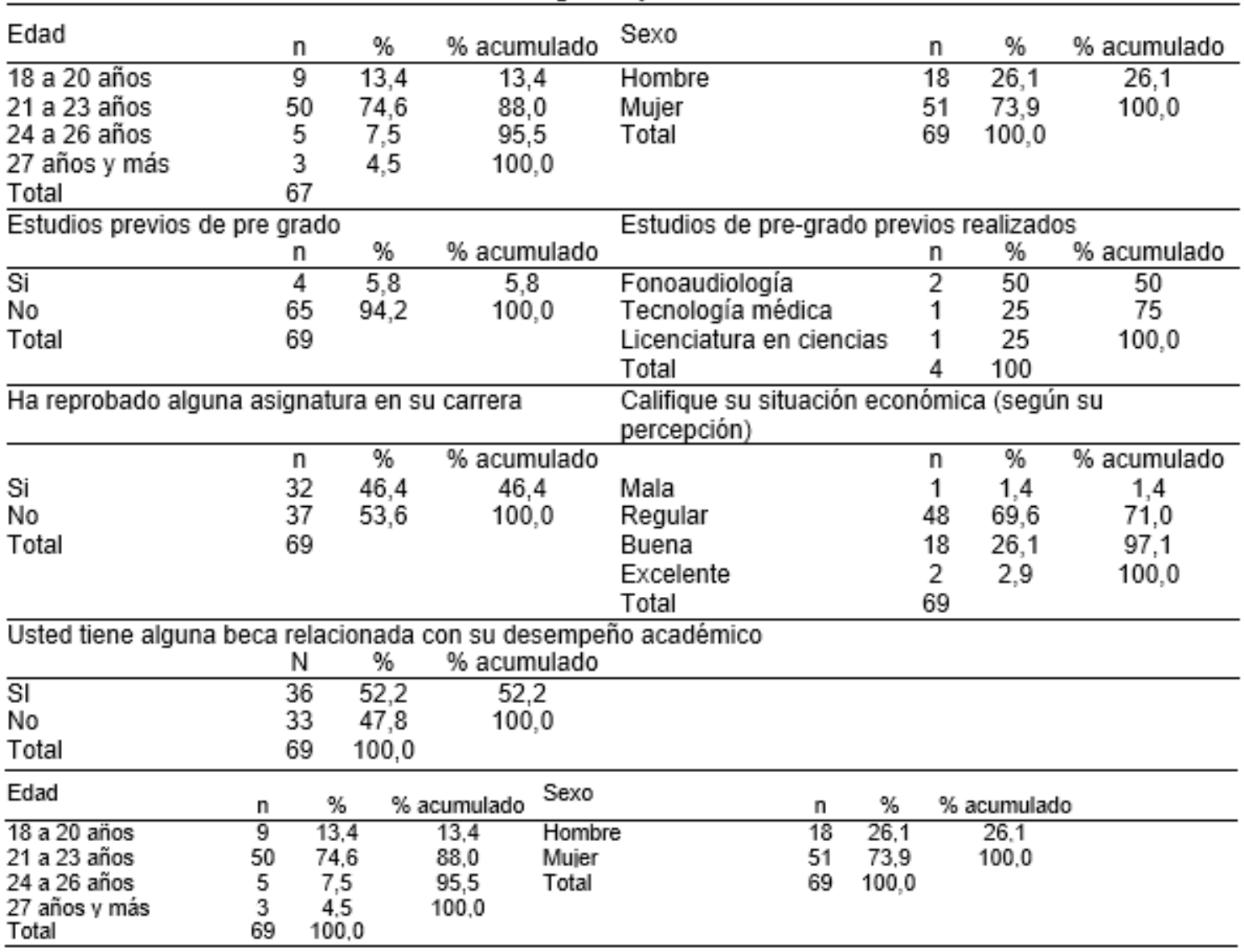


En general, el 74,6\% de los estudiantes de enfermería se ubican en el rango de edad de 21 a 23 años y un 73,9\% son mujeres, además, el 94,2\% no tenían estudios previos de pregrado. De los 4 estudiantes que presentaron estudios previos de pregrado, dos estudiaron Fonoaudiología, uno Tecnología Médica y uno Licenciatura en Ciencias.

Por otra parte, el 53,6\% de los estudiantes no reprobaron ninguna asignatura de su carrera. En lo económico, el 69,6\% de los estudiantes calificaron su situación económica como "regular" y un 52,2\% mantenía alguna beca relacionada con su desempeño académico.

\section{ANÁLISIS DE ESTRESORES}

De la Tabla 3 destaca que a un $84,1 \%$ de los estudiantes de enfermería de tercer año les preocupa mucho y bastante "La relación con el profesor responsable de las prácticas intrahospitalarias", durante la realización de las prácticas, lo cual es corroborado por Timmins y Kaliszer (2002), reconociendo al docente como principal fuente de estrés.

Así mismo, a un 76,8\% de los estudiantes, les preocupa bastante y mucho "Encontrarme en alguna situación sin saber qué hacer”, durante las prácticas clínicas intrahospitalarias.

Tabla 3: Análisis de la relación con los tutores y/o compañeros

\begin{tabular}{|c|c|c|c|c|c|c|}
\hline \multicolumn{4}{|c|}{ La relación con los profesionales de la salud } & \multicolumn{3}{|c|}{ La relación con los compañeros estudiantes de } \\
\hline Categoría & Frecuencia? & & $\%$ acumulado & Frecuencia & $\%$ & $\%$ acumulado \\
\hline Nada & 6 & 8,7 & 8,7 & 10 & 14,5 & 14,5 \\
\hline Algo & 30 & 43.5 & 52,2 & 16 & 23.2 & 37,7 \\
\hline Bastante & 26 & 37,7 & 89,9 & 31 & 44,9 & 82,6 \\
\hline Mucho & 7 & 10,1 & 100,0 & 12 & 17,4 & 100,0 \\
\hline Total & 69 & 100,0 & & 69 & 100,0 & \\
\hline \multicolumn{7}{|c|}{$\begin{array}{l}\text { La relación con el profesor responsable de las prácticas La relación con el/la enfermero/a clínico/a } \\
\text { intrahospitalarias }\end{array}$} \\
\hline Categoría & Frecuencia? & & $\%$ acumulado & Frecuencia & $\%$ & $\%$ acumulado \\
\hline Nada & 5 & 7,2 & 7,2 & 3 & 4,3 & 4,3 \\
\hline Algo & 6 & 8,7 & 15,9 & 25 & 36,2 & 40,6 \\
\hline Bastante & 26 & 37,7 & 53,6 & 31 & 44.9 & 85.5 \\
\hline Mucho & 32 & 46,4 & 100,0 & 10 & 14,5 & 100,0 \\
\hline Total & 69 & 100,0 & & 69 & 100,0 & \\
\hline \multicolumn{4}{|c|}{ No sentirme integrado/a en el equipo de trabajo } & \multicolumn{3}{|c|}{ Encontrarme en alguna situación sin saber qué hacer } \\
\hline Categoría & Frecuencia? & & $\%$ acumulado & Frecuencia & $\%$ & $\%$ acumulado \\
\hline Nada & 16 & 23,2 & 23,2 & 2 & 2,9 & 2,9 \\
\hline Algo & 19 & 27,5 & 50,7 & 14 & 20,3 & 23,2 \\
\hline Bastante & 27 & 39,1 & 89,9 & 33 & 47,8 & 71,0 \\
\hline Mucho & 7 & 10,1 & 100,0 & 20 & 29,0 & 100,0 \\
\hline Total & 69 & 100,0 & & 69 & 100,0 & \\
\hline
\end{tabular}

ESTRÉS POR RELACIONES E IMPLICACIÓN EMOCIONAL DE LOS ESTUDIANTES DE ENFERMERÍA

$\mathrm{Al}$ analizar los reactivos relacionados con el factor implicación emocional del estudiante de enfermería con el paciente durante las prácticas intrahospitalarias (Tabla 4), se observa que a un $79,7 \%$ de los estudiantes les preocupa bastante y mucho "Que mi responsabilidad en el cuidado del paciente sea importante" en las prácticas clínicas intrahospitalarias y que
$81,2 \%$, les preocupa bastante y algo "Que me afecten las emociones del paciente". Por otra parte, a un $78,2 \%$ de los estudiantes de enfermería de tercer año, les preocupa bastante y algo "Tener que estar con un paciente al que se le ha ocultado una mala noticia”.

Los tres puntos anteriores son confirmados por López y Sánchez (2005), como situaciones vividas con más intensidad lo cual es causa de preocupación para los 
alumnos, generando una percepción que es confirmado por Zryewskyj y Davis (1987), quienes comprobaron que el área académica y clínica producía el 78,4\% de los acontecimientos académicos estresantes.

Tabla 4: Implicación emocional con el paciente

\begin{tabular}{|c|c|c|c|c|c|c|}
\hline \multicolumn{4}{|c|}{ Implicarme demasiado con el paciente } & \multicolumn{3}{|c|}{ Que me afecten las emociones del paciente } \\
\hline Válidos & Frecuer & & $\%$ acumulado & Frecuer & & $\%$ acumulado \\
\hline Nada & 12 & 17,4 & 17,4 & 9 & 13,0 & 13,0 \\
\hline Algo & 35 & 50,7 & 68,1 & 28 & 40,6 & 53,6 \\
\hline Bastante & 20 & 29,0 & 97,1 & 28 & 40,6 & 94,2 \\
\hline Mucho & 2 & 2,9 & 100,0 & 4 & 5,8 & 100,0 \\
\hline Total & 69 & 100,0 & & 69 & 100,0 & \\
\hline \multicolumn{4}{|c|}{$\begin{array}{l}\text { Que mi responsabilidad en el cuidado del paciente sea } \\
\text { importante }\end{array}$} & \multicolumn{3}{|c|}{$\begin{array}{l}\text { Tener que estar con un paciente al que se le ha } \\
\text { ocultado una mala noticia. }\end{array}$} \\
\hline Válidos & Frecuer & & $\%$ acumulado & Frecuer & & $\%$ acumulado \\
\hline Nada & 4 & 5,8 & 5,8 & 6 & 8,7 & 8,7 \\
\hline Algo & 10 & 14,5 & 20,3 & 23 & 33,3 & 42,0 \\
\hline Bastante & 28 & 40,6 & 60,9 & 31 & 44,9 & 87,0 \\
\hline Mucho & 27 & 39,1 & 100,0 & 9 & 13,0 & 100,0 \\
\hline Total & 69 & 100,0 & & 69 & 100,0 & \\
\hline
\end{tabular}

ESTRÉS POR FALTA DE COMPETENCIA Y SOBRECARGA EN LA PRÁCTICA CLÍIICA

Respecto de las causas más prevalentes de la falta de competencia, en la Tabla 5 destaca el hecho que a un $84,2 \%$ de los estudiantes de enfermería de tercer año en prácticas clínicas intrahospitalarias, les preocupa mucho y bastante "Meter la pata“, mientras que al $78,3 \%$ de los estudiantes, les preocupa mucho y bastante "Hacer mal mi trabajo y perjudicar al paciente”.

En general, al 75,4\% de los estudiantes les preocupa mucho y bastante "Confundirse de medicación" y al $69,6 \%$ de los estudiantes les preocupa mucho y bastante "Hacer daño físico al paciente", durante las prácticas clínicas intrahospitalarias.

Referido a los tres puntos anteriores, el estudio realizado por Cobo, Carbonell, Rodríguez, Vivo, Castellanos y Sánchez (2010), destacan que la falta de competencia, entre otros, estimulan una percepción elevada de estrés durante las prácticas clínicas.

En relación con la sobrecarga que presentan los estudiantes de enfermería en el transcurso de su práctica clínica intrahospitalaria, se puede inferir que a un $75,4 \%$ de los estudiantes de enfermería de tercer año, les preocupa mucho y bastante la "so- brecarga de trabajo"; y a un $75,3 \%$ de los estudiantes de enfermería les preocupa bastante y mucho "Recibir órdenes contradictorias", lo que es corroborado por Zryewskyj y Davis (1987), quienes confirmaron el hecho que la conjugación del área académica con la clínica producía el 78,4\% de los acontecimientos académicos estresantes.

Finalmente al solicitarles a los estudiantes que realizaran una autopercepción de estrés (Tabla 5) un 89,9\% de los estudiantes se sintió estresado durante sus prácticas clínica intrahospitalarias.

Este último hallazgo ha sido validado por Ferrer, Rojo, Ruiz, Fernández, San Millán y Martínez (2002), quienes afirman que el entorno sanitario suele proporcionar muchos estímulos generadores de estrés debido al contacto con las enfermedades, el dolor, el sufrimiento, la invalidez, la muerte de los pacientes, la necesidad de establecer relaciones con diversos profesionales sanitarios y pacientes, así como por el hecho de tener que desempeñar un nuevo rol para el que aún no están completamente preparados.

Respecto del estrés derivado de la falta de competencia durante las prácticas clínicas intrahospitalarias, a los estudiantes les preocupa mucho y bastante "Meter la pata" y les preocupa mucho y bastante 
"Hacer mal mi trabajo y perjudicar al paciente”, esto dadas las responsabilidades y cargas laborales que genera ser un profesional dentro del área de la salud.

Tabla 5: Análisis de estresores Falta de competencia y sobrecarga

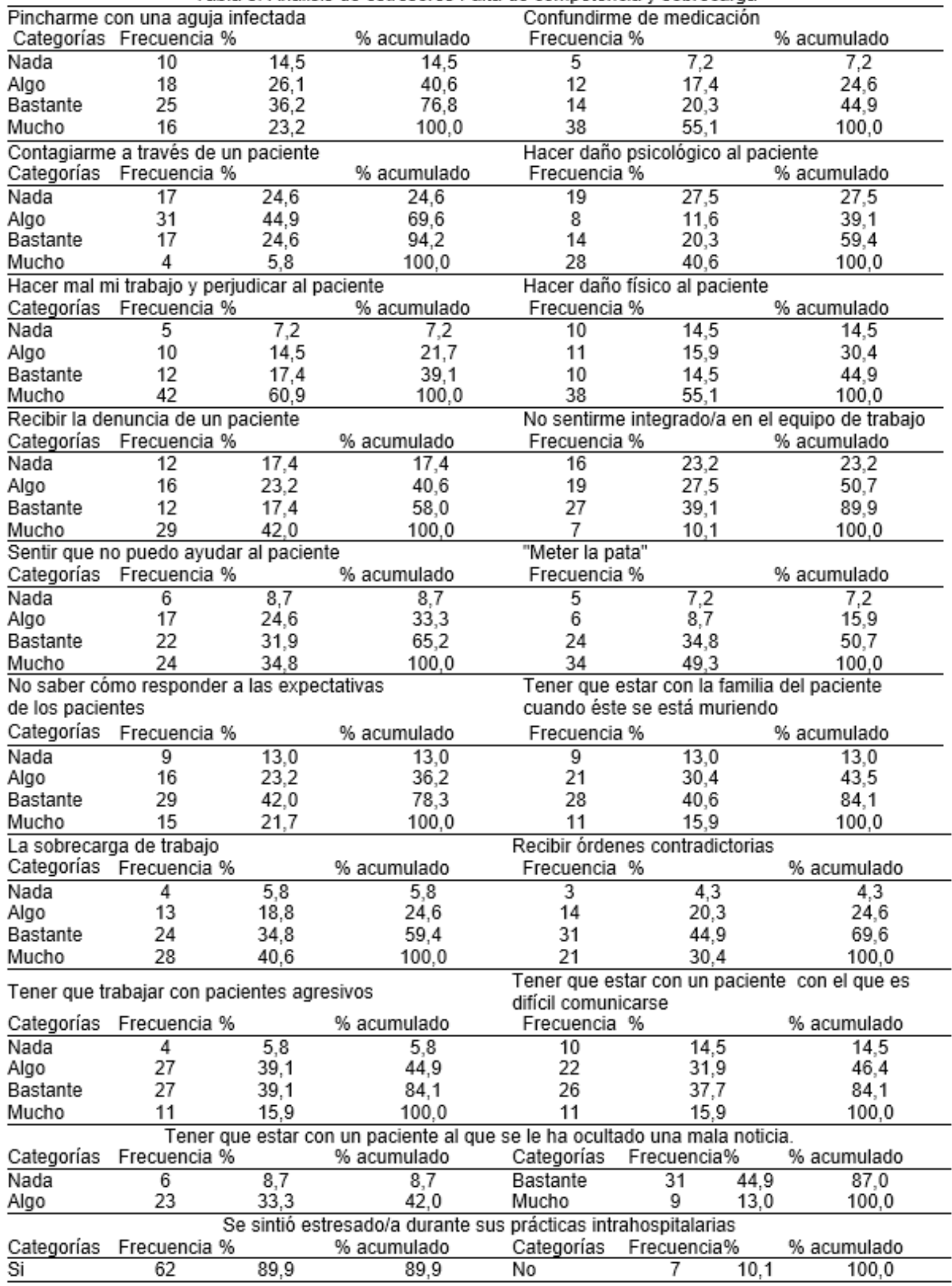


Así mismo, les preocupa mucho y bastante "Confundirse de medicación" así como "Hacer daño físico al paciente", durante las prácticas clínicas intrahospitalarias, puesto que, las consecuencias que genera una mala práctica que atenta contra la integridad física del paciente, es altamente condenada por la sociedad, en razón de lo cual los estudiantes se enfrentan a la presión constante de saber si están haciendo un buen trabajo o si lo podrán hacer bien a futuro.

Finalmente se puede inferir que a más de dos tercios de los estudiantes les preocupa mucho y bastante la "sobrecarga de trabajo" y les preocupa bastante y mucho "Recibir órdenes contradictorias" en las prácticas clínicas intrahospitalarias.

\section{CONCLUSIONES}

Los hallazgos obtenidos en este trabajo permiten orientar las decisiones académicas de formación clínica en enfermería considerando el estrés estudiantil en su multi dimensionalidad. Es preciso tener a la vista además, las posibles consecuencias que un procedimiento mal efectuado por los efectos sobre un paciente y por la tensión a la que se ven enfrentados los estudiantes en práctica clínica.

Así mismo, se considera que cuando los prestadores de servicios de salud enfrentan escenarios críticos con pacientes de alto riesgo, profesionales y practicantes, deben estar preparados para absorber el estrés de la situación, de manera de no alterar sus competencias psicológicas, emocionales e inclusive físicas, que puedan comprometer, en el largo plazo, la propia salud de los prestadores.

Es por esta razón que es determinante encontrar formas en la que los estudiantes de enfermería, que realicen prácticas intrahospitalarias, sean capaces de asimilar las situaciones que enfrentan, manejar el estrés y que finalmente, los denominados estresores emocionales, no terminen por afectar directamente la calidad de vida laboral y bienestar de los estudiantes en práctica clínica.

\section{REFERENCIAS}

Basso, L; Ardiles, B; Bernal, M; Cánovas, M; González, C; Kroff, M; \& Soto, A. (2008). Factores derivados de los laboratorios intrahospitalarios que provocan estrés en los estudiantes de enfermería. Revista Latino-Americana de Enfermagem, 16(5), 805-811. Recuperado el 18 de octubre de 2016, de http://www. scielo.br/scielo.php?script=sci_arttext\&pid=S0104-11692008000500002\&lng=en\&tlng $=$ es . 10.1590/So10411692008000500002 .

Bruno Selye H. Tensión sin Angustia. Madrid: Guada: Guadarrama; 1975.

Burgos, 2004. Calidad de vida y estrés de los estudiantes de la escuela de Enfermeria, Universidad Austral de Chile, 2 semestre académico 2003).

Cannon, W.B. (1929). Bodily changes in pain, hunger, fear and rage ( $2^{\underline{a}}$ ed.). Nueva York: Appleton.

Celis, J, Bustamante, M, Cabrera, D, Cabrera, M, Alarcón, W y Monge, E. (2001). Ansiedad y Estrés Académico en Estudiantes de Medicina Humana del Primer y Sexto Año.

Cobo, A, Carbonell, R, Rodríguez, C, Ortega, I, Castellanos, R, Sánchez, A. (2010). Estresores y ansiedad de los estudiantes de enfermería en sus primeras prácticas clínicas. Nure Investigación, № 49 .

Collados, J, García, J, (2012). Riesgo de estrés en estudiantes de Enfermería durante las prácticas clínicas. Revista Científica de Enfermería. $\mathrm{N}^{\circ} 4$.

Da Silva, M. (2006). Nuevas perspectivas de la Calidad de Vida Laboral y sus relaciones con la eficacia organizacional. Tesis Doctoral, Universidad de Barcelona, España.

Davis, K., \& Newstrom, J. (1993). Comportamiento humano en el trabajo. México: Mc Graw-Hill.Octava edición.

Eyssautier M. 2006. Metodología de la investigación. México D.F.: Editorial Thomson; 2006.

Ferrer Pascual MaA, Rojo Pascual MaC, Ruiz Gómez MaC, Fernández Araque AM, San Millán MG, y Martínez León, JC (2002). Análisis situacional de las prácticas clínicas en la Escuela de Enfermería de Soria. Metas Enferm, ; 5 
(44): 18-22.

García-Moran, M. C. y Gil-Lacruz, M. (2016). El estrés en el ámbito de los Profesionales de la Salud. Universidad de Zaragoza, Página 19, enero-diciembre. Pp. 11-30. Zaragoza, España. ISSN 15606139.

Gil-Lacruz, M. (2007). Psicología social y bienestar. Zaragoza: Prensas Universitarias.

González, M. C. (2017). Satisfacción Laboral del profesional de Enfermería del Servicio de Emergencias del Hospital “José Casimiro Ulloa" 2015. Tesis de Especialización. Universidad Nacional Mayor de San Marcos. Lima, Perú. Obtenido de: http://cybertesis.unmsm. edu.pe/bitstream/handle/cybertesis/7104/Gonzales_mm.pdf?sequen$\mathrm{ce}=1$ \&isAllowed $=\mathrm{y}$

Granados, I. (2011). Calidad de Vida Laboral: Historia, Dimensiones y Beneficios. Revista IPS, Facultad de Psicología, Universidad Nacional Mayor de San Marcos. Vol. 14, N"2, pp 271-276. Lima, Perú.ISSN 1609-7445.

Hernández R. , Fernández C. y Baptista P. (2006). Metodología de la Investigación (4.a ed.) McGraw-Hill, México D. F. Retrieved from https://goo.gl/mRZLii

Holmes TH, RaheRH. The Social readjustment ratings scale. J Psychosan Res 1967; 11: 213-218.

Holmes, T.H. y Rahe, R.H. (1967). The social readjustment rating scales. Journal of Psychosomatic Research, 11, 213218.

Lázarus R, Folkman S. (1986). Estrés y procesos cognitivos. Ediciones Martínez Roca.

Lazarus, R.S. y Folkman, S. (1984). Stress, appraisal and coping. Nueva York: Springer. (Traducción, Barcelona, Martínez Roca, 1986).

López V, Francisca, \& López M, María José. (2011). Situaciones generadoras de estrés en los estudiantes de enfermería en las prácticas clínicas. Ciencia y enfermería, 17(2), 47-54. Recuperado en 20 de noviembre de 2016 , de http://www.scielo.cl/scielo.php?script=sci_arttext\&pi$\mathrm{d}=$ So717-95532011000200006\&lng=es\&tln- $\mathrm{g}=\mathrm{es} .10 .4067 /$ So717-95532011000200006

López, I. Sánchez, V. (2005). "Percepción del estrés en estudiantes de enfermería en las prácticas clínicas”. Publicado en Enfermería Clínica. vol.15 núm. o6.

Mahat G. Stress and coping: first year Nepalese nursing students in clinical settings. J Nurs Educ. 1996; 35(4):163-9.

Martín M, Isabel (2007). Estrés académico en estudiantes universitarios, Recuperado el 30 de marzo de 2017, http://www. apuntesdepsicologi.es/index.php/revista/article/viewFile/117/119

Moscoso, S. Estrés, salud y emociones: estudio de la ansiedad, cólera y hostilidad. Revista de psicología vol. $3 \mathrm{n}^{\circ}$ 3julio 1998.

Pérez, C; Alameda, A; Albéniz, C. (2002) La formación práctica en enfermería en la Escuela Universitaria de Enfermería de la Comunidad de Madrid. Opinión de los alumnos y de los profesionales asistenciales: Un estudio cualitativo con grupos de discusión. Rev. Esp. Salud Pública. Madrid, v. 76, n. 5, Recuperado el o5 noviembre 2016 de <http://scielo.isciii.es/scielo.php?script=sci_arttext\&pi$\mathrm{d}=$ S1135-57272002000500013\&lng=es\&nr$\mathrm{m}=\mathrm{iso}>$.

Selye, H. (1960). La tensión en la vida. Buenos Aires: Cía. Gral. Fabril.

Selye, H. (1976). Stress without Distress. Psychopathology of Human Adaptation, 137-146. doi:10.1007/978-1-4684-2238-2_9

Timmins F, Kaliszer M. Aspectos de los programas de educación de enfermería que frecuentemente causan estrés a los estudiantes de enfermería- encuesta de muestreo de hallazgos. Enfermera Educ Hoy. 2002; 22: 203 - 211.

Zryewskyj T, Davis L. Sources of stress in third year baccalaureate nursing students. AARN-News-Lett. 1987; 43(3):24-5.

Zupiria Gorostidi, X y otros. (2002). Cuestionario bilingüe de estresores de los estudiantes de enfermería en las prácticas clínicas. Gaceta Sanitaria, V17; o1; 37-51

Zupiria, X, Uranga, J, Alberdi, J, y otros, (2006). Fuentes de estrés en la práctica clínica de los estudiantes de enfermería. Evolución a lo largo de la diplomatura. Enferm Clin. ; 16(5):231-7. 